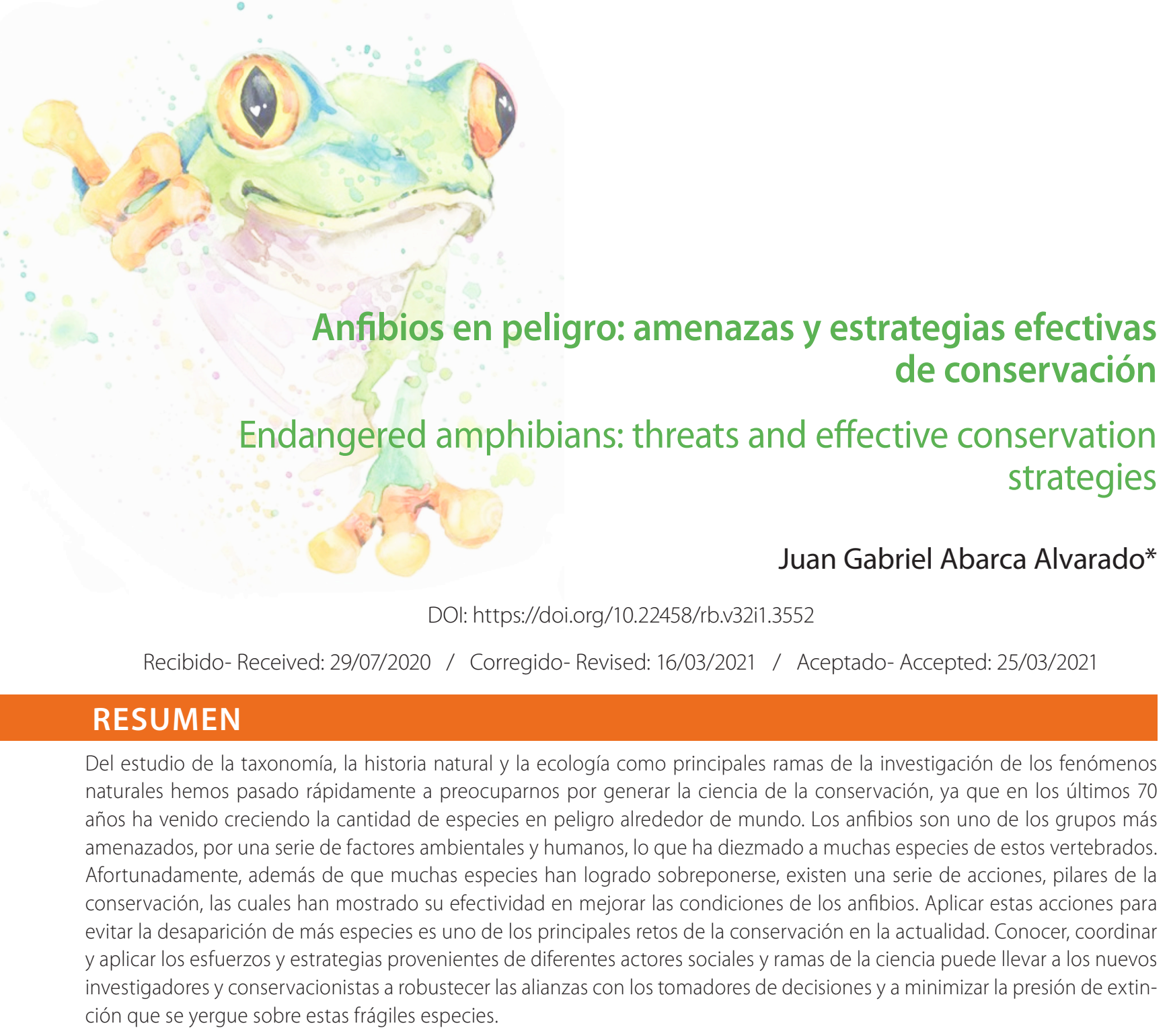

Palabras clave: pérdida de biodiversidad; anfibios; extinción; conservación; sinergia.

\title{
ABSTRACT
}

From taxonomy, natural history, and ecology as the main fields of study of natural phenomena, we have quickly moved to concern to generate a conservation science due to the worldwide significant increase in the number of endangered species over the last 70 years. Amphibians are one of the most threatened groups because of a series of environmental and human factors, which have decimated many species of these vertebrates. Fortunately, in addition to the fact that many species have managed to overcome the threats, there are a series of actions, pillars of conservation, which have shown their effectiveness at improving the conditions of amphibians. Carrying out these actions to avoid the disappearance of more species is one of the main challenges of conservation today. Knowing and coordinating efforts, in addition to applying strategies that come from different social actors and branches of science can lead new researchers and conservationists to strengthen alliances with decision-makers to minimize the extinction pressure on these fragile species.

Key words: biodiversity loss; amphibians; extinction; conservation; synergy.

* Laboratorio de Recursos Naturales y Vida Silvestre, Escuela de Ciencias Biológicas, Universidad Nacional, Heredia, Costa Rica; barcazajuan@gmail.com

ID: https://orcid.org/0000-0002-1261-4400 


\section{Anfibios y biodiversidad}

Es posible que muchos de los lectores se hayan dado cuenta que nosotros, como seres humanos, utilizamos los recursos naturales de forma excesiva, sin pensar que esto, consecuentemente, puede disminuir su disponibilidad para otras especies animales. Nuestro modelo de desarrollo vigente, altamente globalizado e industrializado, ha provocado que muchos de los sistemas biológicos más frágiles, como los arrecifes de coral o las extensas selvas tropicales, se deterioren paulatinamente y con ello empiecen a perder gran parte de su biodiversidad (Altieria et al., 2017; Giam, 2017).

¿Por qué nos debería preocupar esa pérdida de biodiversidad? Aunque se podrían expresar muchos motivos por los cuales nos deberíamos preocupar, el que nos compete para la presente lectura tiene que ver con las desapariciones repentinas de muchas especies, llamadas también "extinciones masivas". En la larga historia de la tierra han ocurrido cinco grandes extinciones y muchos proclamamos que estamos viviendo la sexta gran extinción masiva (cuadro 1). Después de eventos de extinción masiva los ecosistemas naturales se reacomodan, cambian, se renuevan y la biodiversidad que existía puede ayudar a mitigar su impacto, mientras más especies existan, más tendrán oportunidad de sobrevivir y adaptarse a las nuevas condiciones del ambiente, ocupando los espacios que dejan aquellos grupos desaparecidos (Molles, 1999).

La biodiversidad (cantidad de especies de animales y plantas de un sitio) no sólo es importante por lo mencionado anteriormente, sino porque brinda muchos beneficios actuales para la vida en el planeta. El trópico americano representa una de las regiones geográficas con mayor biodiversidad en el mundo, es una región ideal para estudiar las causas y consecuencias de la pérdida de especies, procesos de sucesión y ejemplos de

\begin{tabular}{|c|c|c|c|c|}
\hline \multicolumn{5}{|c|}{$\begin{array}{c}\text { Cuadro } 1 \\
\text { Las principales extinciones masivas en la tierra }\end{array}$} \\
\hline Período & $\begin{array}{l}\text { Millones } \\
\text { de años atrás }\end{array}$ & $\begin{array}{l}\text { Pérdida de } \\
\text { especies }\end{array}$ & Posibles causas & Especies desaparecidas \\
\hline Ordovícico & 440 & $85 \%$ & $\begin{array}{l}\text { Capa de hielo, cambio climático, caída en el nivel } \\
\text { del mar. }\end{array}$ & $\begin{array}{l}\text { Trilobites, braquiópodos y } \\
\text { graptolites. }\end{array}$ \\
\hline Devónico & 359 & $82 \%$ & $\begin{array}{l}\text { El mar se quedó sin oxígeno, cambios en el nivel del } \\
\text { mar, impacto de asteroides y cambio climático. }\end{array}$ & Peces e invertebrados marinos. \\
\hline Pérmico & 246 & $96 \%$ & $\begin{array}{c}\text { Asteroides, erupciones volcánicas, inundación } \\
\text { de basalto y una caída drástica en los niveles de } \\
\text { oxígeno. }\end{array}$ & $\begin{array}{l}\text { Reducción en todas los grupos } \\
\text { conocidos, incluidos muchos } \\
\text { tipos de insectos. }\end{array}$ \\
\hline $\begin{array}{l}\text { Triásico- } \\
\text { Jurásico }\end{array}$ & 200 & $76 \%$ & $\begin{array}{l}\text { Cambio climático, actividad volcánica y un impacto } \\
\text { de un asteroide. }\end{array}$ & $\begin{array}{l}\text { Arcosaurios, reptiles marinos y } \\
\text { anfibios gigantes. }\end{array}$ \\
\hline $\begin{array}{l}\text { Cretácico- } \\
\text { Terciario }\end{array}$ & 65 & $76 \%$ & Posible impacto de un asteroide. & $\begin{array}{l}\text { La mayoría de especies de } \\
\text { dinosaurios }\end{array}$ \\
\hline Holoceno & 11 mil años & ¿? & Cambio climático, acciones humanas. & $\begin{array}{l}\text { Muchas especies de plantas, } \\
\text { animales, anfibios, mamíferos, etc }\end{array}$ \\
\hline
\end{tabular}

Nota. Modificado de Barnosky et al., 2011. 
resiliencia ecológica, ya que a pesar de su alta biodiversidad han ocurrido pérdidas considerables de especies dentro de áreas naturales protegidas, y son principalmente los anfibios el grupo que más ha perdido su diversidad en los últimos años (Stuart et al., 2004).

Los anfibios son un grupo de animales muy importantes para mantener los ecosistemas naturales con buena salud (aunque a simple vista no lo parezca). Por ejemplo, las ranas y sapos se utilizan como indicadores de la salud de los ecosistemas ya que durante su vida la mayoría de las especies de anfibios pasa de un hábitat acuático a uno terrestre (cuadro 2, fig. 1), además son muy dependientes de temperaturas cálidas, lluvia y humedad abundante; si no existe suficiente disponibilidad de agua pasan hibernando o estibando durante largos períodos de invierno o sequías (Wells, 2007). Las larvas o renacuajos tienen vida acuática y son omnívoros, mientras que los adultos habitan ecosistemas acuáticos o terrestres y son depredadores; por todo ello, los anfibios cumplen distintos papeles dentro del intercambio de nutrientes y energía en las complejas redes naturales de la vida, llamadas redes tróficas (Whiles et al., 2006).

Los anfibios adultos poseen una piel muy permeable, la cual permite el intercambio gaseoso con el medio,así también es muy permeable al agua y fácilmente puede perderla por desecación, aunque no es uniforme en todo el cuerpo, ya que varía en grosor, vascularización, permeabilidad y en su respuesta a las hormonas (Wells, 2007). Además, la textura varía entre especies, muchas poseen tubérculos muy vascularizados para la absorción de agua y la mayoría de ranas presenta modificaciones de comportamiento que les permiten escoger el hábitat adecuado para evitar una excesiva perdida de agua (Wells, 2007).
Figura 1

Generalización de las etapas de desarrollo en anfibios

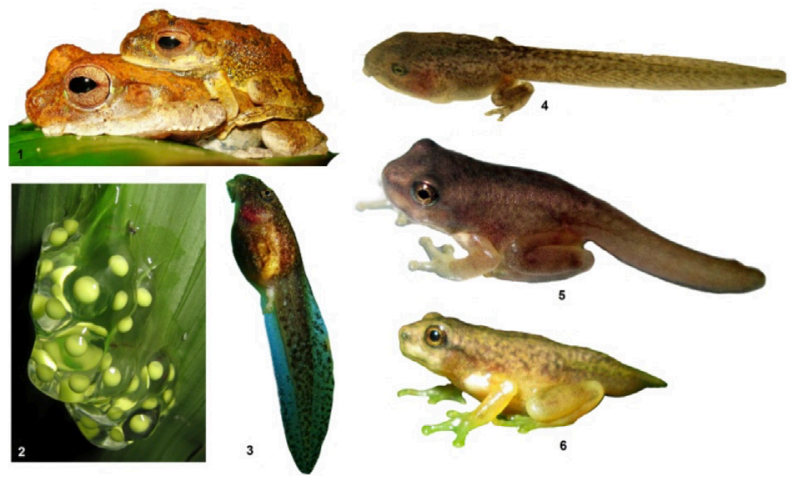

Cuadro 2

Etapas del desarrollo en anfibios

y su dependencia al agua

1. La reproducción ocurre en época lluviosa, cerca de ríos o en ambientes con suficiente humedad.

2. Para las especies que ponen huevos, lo hacen en una masa gelatinosa que necesita mantenerse húmeda.

3. Las larvas están adaptadas a una vida acuática.

4. La metamorfosis es el cambio de la vida acuática a la vida terrestre.

5. Los metamorfos no se alimentan mientras pasan al ambiente terrestre.

6. Durante las primeras semanas los juveniles son más vulnerables a amenazas ambientales.

Nota. Wells, 2007.

La piel es el órgano más importante de este grupo de animales, de allí su gran dependencia del agua, la lluvia, las zonas inundadas, los bosques lluviosos y nubosos tropicales. En general siempre están relacionados a zonas con mucha humedad y mientras mayor sea el régimen de lluvias más diverso será el grupo. Sin embargo, la permeabilidad de la piel provoca que muchas especies de anfibios sean más susceptibles a sustancias contaminantes dispersas en cuerpos de agua o en el ambiente en general, cambios drásticos de 
temperatura o enfermedades ambientales (Lebboroni et al., 2006).

\section{Principales amenazas hacia los anfibios}

Hasta hace tan solo 40 años, los anfibios tenían tantos problemas como podrían tener el resto de animales, sin embargo debido a una serie creciente de acontecimientos desfavorables, su estudio se ha convertido en una ciencia "de urgencia", en donde ya no solo se deben dedicar esfuerzos a entender su interesante historia natural, sino también a evitar que desaparezcan. Actualmente, es pertinente incluir en el estudio de los anfibios las amenazas que los califican como un grupo en alto riesgo de desaparecer. Por tanto, su conservación ha llegado a ser un tema muy importante en los últimos 30 o 40 años, lo cual brinda a nuestra generación la oportunidad y el compromiso de estudiar el fenómeno de su extinción en primer plano.

Los anfibios son los vertebrados que más han sufrido la pérdida de diversidad alrededor del mundo; cerca de $41 \%$ de todas las especies, cuya categoría de conservación ha sido analizada, están en peligro de extinción (IUCN, 2020). Entre el año 1980 y el año 2000, muchísimas especies de ranas desaparecieron de sus localidades conocidas y en muchas otras hubo una considerable disminución poblacional (Bolaños, 2009). Actualmente, se consideran extintas o posiblemente extintas 35 especies de anfibios, mientras que 2200 especies están bajo alguna categoría de amenaza (IUCN, 2020).

Se mencionarán diversos factores que podrían ser los causantes de este declive poblacional mundial de anfibios (figs.2-3). El entendimiento del fenómeno se torna bastante más complejo cuando se habla de efectos sinérgicos (en conjunto) entre los factores. La permeabilidad de su piel hace a los anfibios muy sensibles al aumento de la temperatura, las radiaciones, los contaminantes y las infecciones dérmicas (Kerby, Hart, y Storfer, 2012). Es posible que algunos efectos como el calentamiento global, la contaminación, la deforestación, el tráfico internacional de especies o factores aún desconocidos estén actuando de forma sinérgica para crear un escenario ideal para la aparición de nuevas enfermedades letales (Rohr et al., 2008), llevandoa muchas especies de anfibios al borde de la extinción (Pounds et al., 2006; Van Rooij et al., 2015, Whitfield et al., 2016).

Modificación de hábitat: El factor incluye la deforestación, el cambio en el uso de la tierra para agricultura y el urbanismo. Lo anterior es una evidente amenaza ya que los anfibiospierden su hábitat, sus áreas de reproducción, el alimento, el refugio; todo esto produce un claro descenso de la diversidad, quedando solo pocas especies que pueden soportar los drásticos cambios. Este factor produce a su vez una fragmentación del hábitat que provoca un aislamiento de las especies en pequeños territorios naturales.

Introducción de especies exóticas: Factor que se presenta por la introducción de peces (tilapia o trucha principalmente) que pueden alimentarse vorazmente de huevos o renacuajos de anfibios, también ranas exóticas (rana toro norteamericana, rana coquí) que pueden afectar tanto directamente por competición como indirectamente por transportar enfermedades infecciosas o camarones de río (también vector de enfermedades), entre otros. 


\section{Figura 2}

Amenazas hacia los anfibios
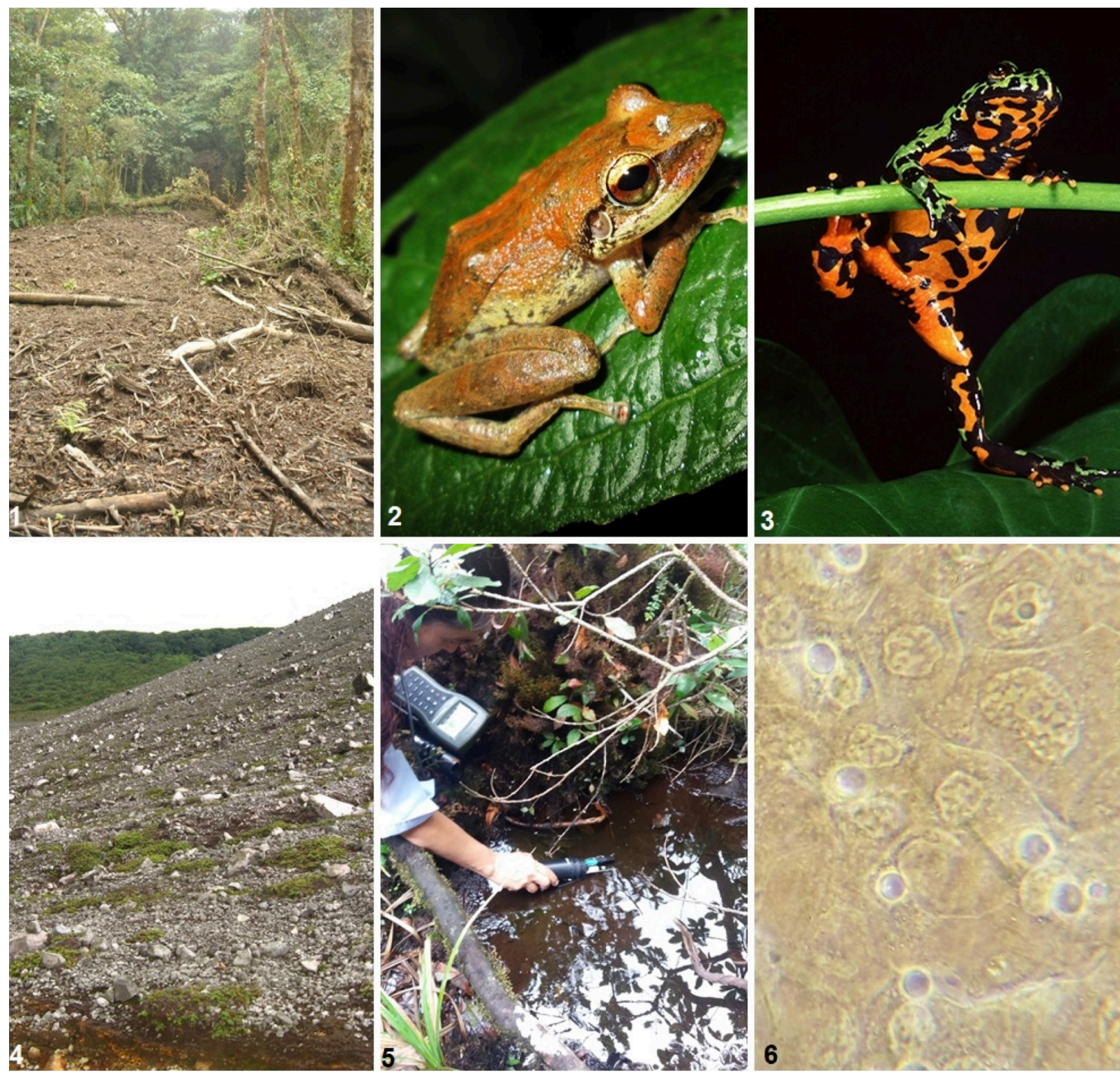

Nota. 1. Modificación del hábitat, 2. Especies exóticas, 3. Comercio, 4. Cambio climático, 5. Contaminantes, 6. Enfermedades. Fotografías: 1-2,4-6: Juan Abarca, 3: RealMagick

Sobreexplotación o comercio: Producto de la exportación ilegal al extranjero de especies nativas (debido al comercio de especies para mascotas, legal o ilegal). También provoca la introducción de especies exóticas en nuevos hábitats cuando se liberan o se escapan, lo cual se relaciona con el factor de amenaza anterior.
Cambio climático: Aún no está claro en qué medida esta amenaza puede afectar a los anfibios, pero es posible quela disminución de las lluvias, el aumento de los años secos, la menor formación de nubes en zonas montañosas, los aumentos de temperatura y el aumento de radiación UV, todos factores relacionado con cambios drásticos y rápidos en el clima de la 
tierra, pueden afectar mucho a especies que dependen de patrones regulares de lluvias y temperatura para vivir y reproducirse.

Figura 3

Presión de las amenazas sobre la extinción de los anfibios

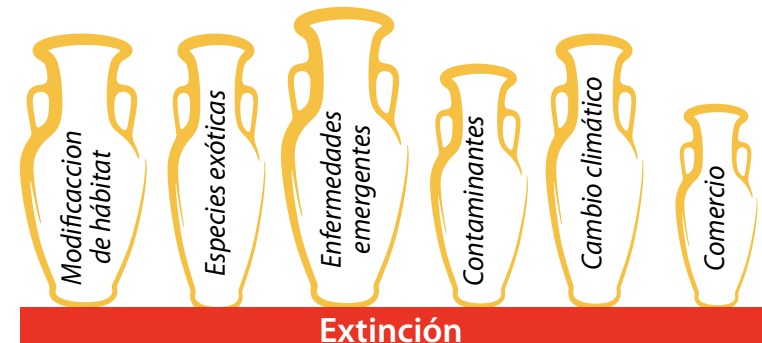

Extinción

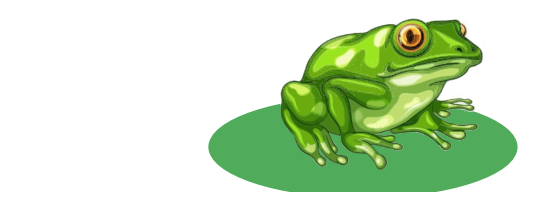

Nota. Basado en Sutherland et al., 2019.

Contaminantes: Los pesticidas, herbicidas, fungicidas, etcétera, se transportan a los sitios reproductivos de los anfibios y han demostrado tener efectos letales para poblaciones de anfibios en muchas partes del mundo. El impacto en los anfibios por el uso excesivo de estos químicos en la agricultura no ha sido bien evaluado; no obstante, la piel de estos animales sirve como absorbente de los químicos presentes en el agua o el ambiente y los hace mucho más vulnerables, produciéndoles ya sea la muerte, deformidades, alteraciones reproductivas, entre otros males.

Enfermedades emergentes: Debido a la globalización y al comercio internacional de especies es más probable que nuevos patógenos lleguen a muchas partes del mundo. La aparición de enfermedades infecciosas se relaciona con muertes masivas de anfibios alrededor del mundo, específicamente con una enfermedad emergente conocida como quitridiomicosis, producida por un microhongo, la cual afecta severamente la piel de los anfibios (fig. 4). Se considera que es la peor enfermedad infecciosa jamás registrada entre los vertebrados en términos del número de especies afectadas y llevadas a la extinción (Wren et al., 2015).

La quitridiomicosis no es la única enfermedad que puede afectar a los anfibios, ya que existen otras enfermedades infecciosas que han sido poco estudiadas. Tanto el cambio climático como la presencia de contaminantes son factores que pueden debilitar a los anfibios, disminuir su sistema inmune y facilitar así la infección por patógenos. Como vemos, todas las amenazas mencionadas pueden coadyuvar a generar una mayor presión de extinción sobre los anfibios, llevando a muchas especies a disminuir sus poblaciones o desaparecer por completo.

\section{Estrategias de conservación}

Sean cuales fueren las causas y las amenazas que estén afectando a los anfibios, es un hecho que muchas poblaciones de ranas han desaparecido, como también es un hecho alentador que muchas otras, aparentemente desaparecidas, se han vuelto a encontrar en muchos lugares. A partir del año 2000, en varias partes del mundo ha habido una gran cantidad de reportes de especies de anfibios que han sido observados de nuevo después de pasar un lapso de 30-40 años sin haberse observado (Whitfield et al., 2016), las causas de su desaparición temporal y actual sobrevivencia, aún se siguen debatiendo.

El saber por qué algunas especies aparentemente diezmadas han logrado sobreponerse es un tema sumamente importante para la conservación; sin embargo, no hemos logrado terminar de entender las causas de las desapariciones, y debemos empezar a realizar 
Figura 4

Proceso de infección del hongo quitridio que produce la quitridiomicosis en los anfibios y como evitar su dispersión

\section{ASÍ AFECTA EL HONGO QUITRIDIO A LAS RANAS}

\section{El quitridio}

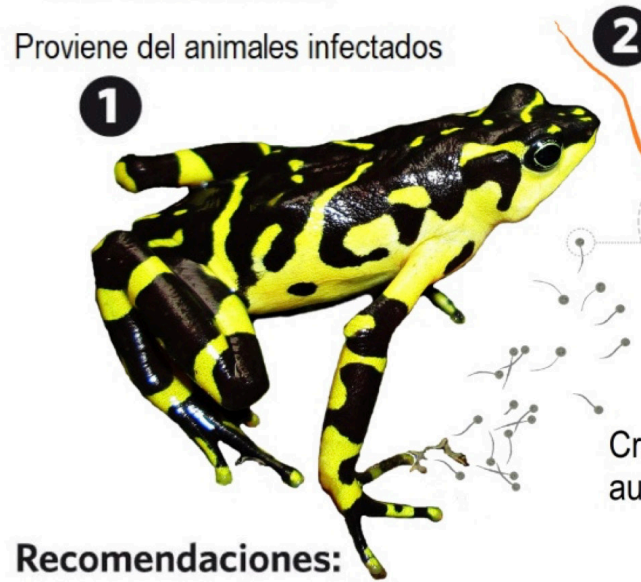

- Evitar trasportar e introducir ranas en nuevos lugares.

- Lavar equipo y calzado de montaña con cloro o agua caliente.

- Evitar la manipulación de cualquier rana si no es parte de un estudio.
Se aloja en las capas superficiales de la piel

Crece y se reproduce aumentando la infección

\section{3}

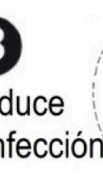

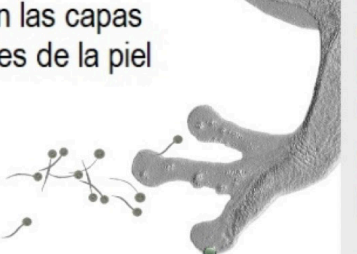

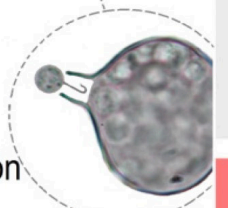

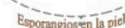

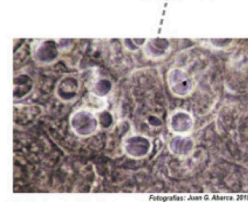

\section{Signos}

Excesivo desprendimiento de piel.

Decoloración y

ulceración de la piel.

Movimientos lentos y

posturas inusuales.

\section{Efectos}

Desequilibrio en la absorción de sales lo que produce un paro cardíaco.

Afecta más a ranas con bajas defensas inmunológicas, que sufren estrés y en individuos juveniles. conjeturas sobre las causas de la resistencia de algunas especies. Sin duda alguna, especies capaces de tolerar de cierta manera los embates de cada uno de los factores que las amenazan, las cuales representan especies sobrevivientes a una reciente presión de selección natural.

Muchas de las especies sobrevivientes pueden haber desarrollado en su organismo algunas formas para adaptarse a la presencia de las amenazas, bien por la mejoría de su sistema inmunológico que le ha permitido combatir las enfermedades, o por permanecer en poblaciones separadas o en ambientes más adecuados, llamados "refugios climáticos", así como por cambios en sus hábitos y comportamientos o simplemente porque han pasado desapercibidos de los ojos de los investigadores durante largo tiempo.
La capacidad de sobrevivencia de una especie es un evento en gran parte azaroso, influenciado por las condiciones ambientales específicas y por la capacidad interna de cada especie de poder cambiar ante dichas condiciones. Algunas especies indudablemente no van a lograr sobreponerse a las amenazas, pero aquellas que sí, nos pueden ayudar a entender toda esta dinámica compleja de los cambios en la biodiversidad de los ecosistemas.

La dicha de poder observar una especie amenazada que se creía desaparecida es, sin duda alguna, un motivo de gran alegría, y el mostrar al mundo el redescubrimiento alienta la esperanza de que muchas más especies puedan superar la crisis global de los anfibios. Pero una vez que la emoción y la cobertura de los medios han pasado, ¿qué queda?, ¿qué 
hacemos con estas especies que han sido encontradas nuevamente? Cabe preguntarse, ¿es necesario dirigir los esfuerzos a buscar más especies desaparecidas?, o por el contrario ¿debemos volcar los recursos a proteger las que ya reaparecieron o parecen volver a incrementar sus poblaciones? Por una parte, mientras más especies amenazadas estén en un lugar, mayor protección al sitio se puede solicitar a las autoridades, sin duda "gran cantidad de especies recuperadas" significa muy buenas noticias. Sin embargo, muchas de estas observaciones se han presentado con pocos individuos, las poblaciones siguen estandomuy bajas si se comparan con reportes previos a las desapariciones. Por tanto, el esfuerzo por evitar más pérdidas debe combinarse con la conservación de las ya existentes.

En este punto, el lector puede hacerse la pregunta, que probablemente es la misma que se haría la comunidad científica: ¿cuáles especies proteger y cómo hacerlo? Gracias a una excelente recopilación de información a nivel mundial la Unión Internacional para la Conservación de la Naturaleza (IUCN) nos brinda las pautas de conservación por seguir y nos indica cuáles especies deberían de recibir más atención en la conservación. Las listas rojas de las especies amenazas en el grupo de anfibios se mantienen actualizadas y gracias a ello podemos saber el rating de las especies más afectadas por región, por país y por grado de amenaza (fig. 5).

Las especies con mayor grado de amenaza actualmente se catalogan como "en peligro crítico", son aquellas que sabemos no han desaparecido, pero sus poblaciones son muy bajas y, por tanto, son las que deberían recibir mayor esfuerzo de conservación, mientras que las catalogadas como extintasson aquellas que después de su desaparición, hasta la actualidad, no se han vuelto a observar; con ellas los esfuerzos y recursos deberían ser mayores para poder contar con búsquedas más exhaustivas. Asimismo, en cuanto a las otras especies con una categoría de amenaza menor, los esfuerzos se deben enfocar en conservar el hábitat y estudiar su dinámica poblacional, para evitar que suban a una categoría de mayor riesgo.

\section{Figura 5}

Número de especies de anfibios según su categoría de amenaza

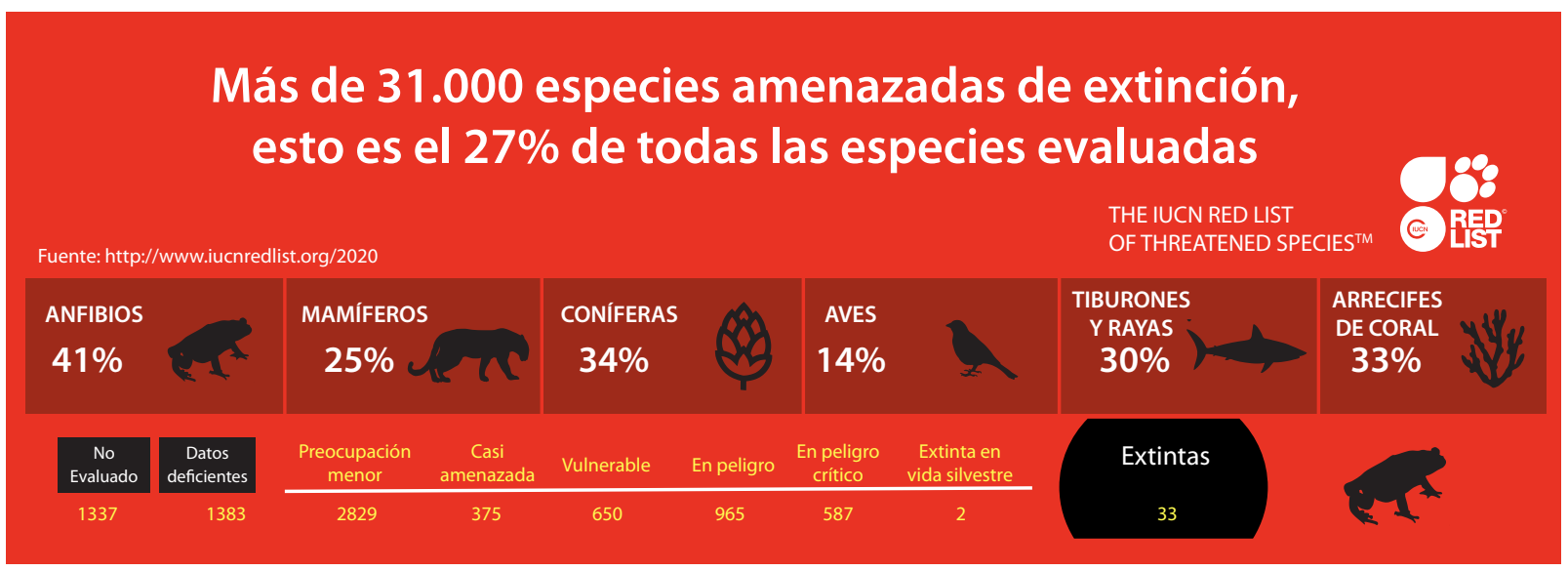

Nota. IUCN, 2020. 
Figura 6

Acciones de conservación
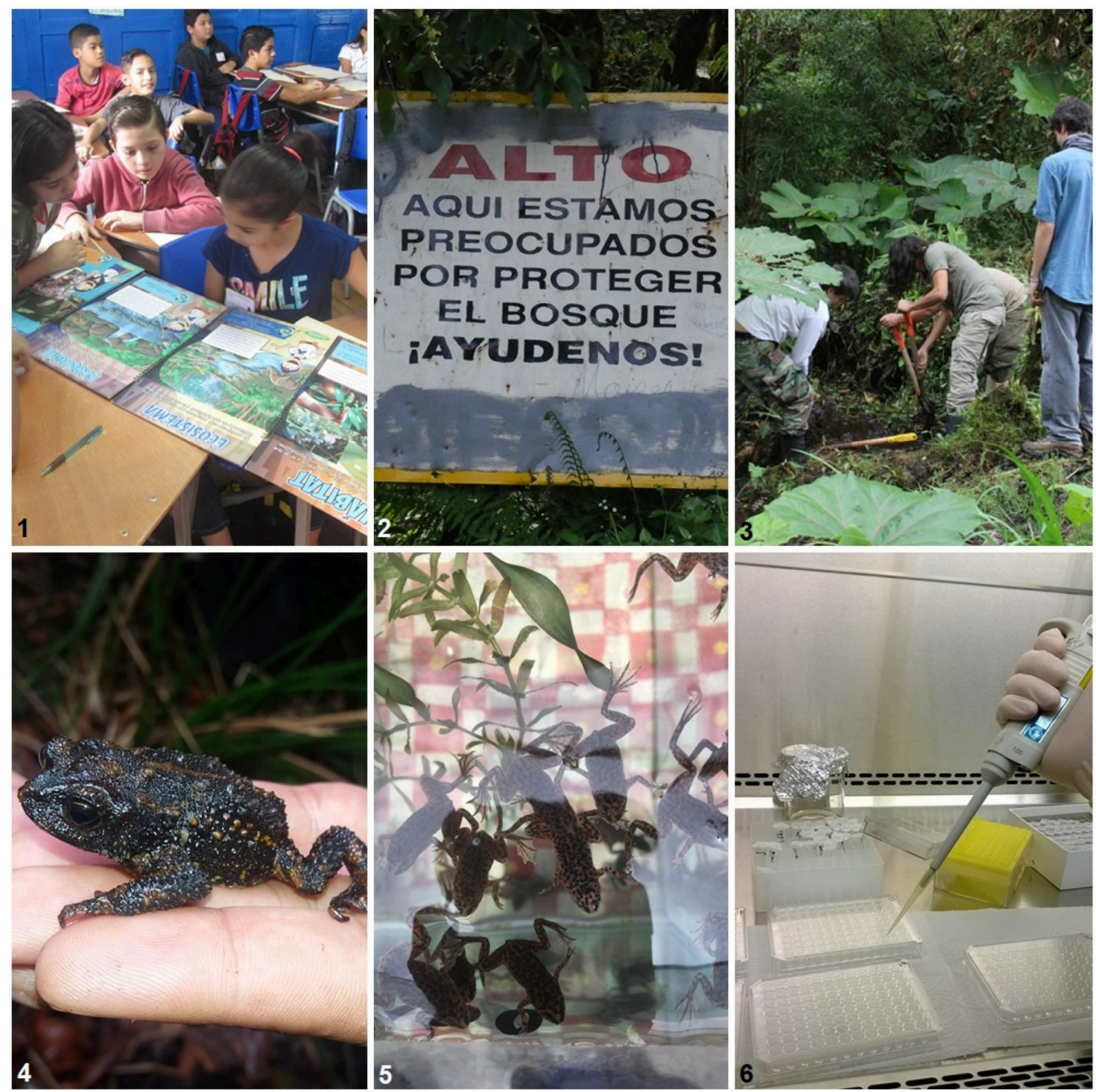

Nota. 1. Educación ambiental, 2. Protección de tierras, 3. Creación de hábitats, 4. Mejora en la legislación, 5. Remoción de especies invasoras, 6. Investigación. Fotografías: 1-4,6: Juan Abarca, 5: Randall Jiménez.

Para responder la pregunta del ¿cómo proteger a estos anfibios amenazados?, existen acciones y estrategias que han demostrado ser eficientes para aumentar las poblaciones de anfibios o han tenido una repercusión favorable en la recuperación de algunas especies de anfibios (Sutherland et al., 2019). Las acciones o pilares de la conservación de anfibios (figs. 6-7) son los siguientes: 
Figura 7

Amenazas y pilares de la conservación en los anfibios

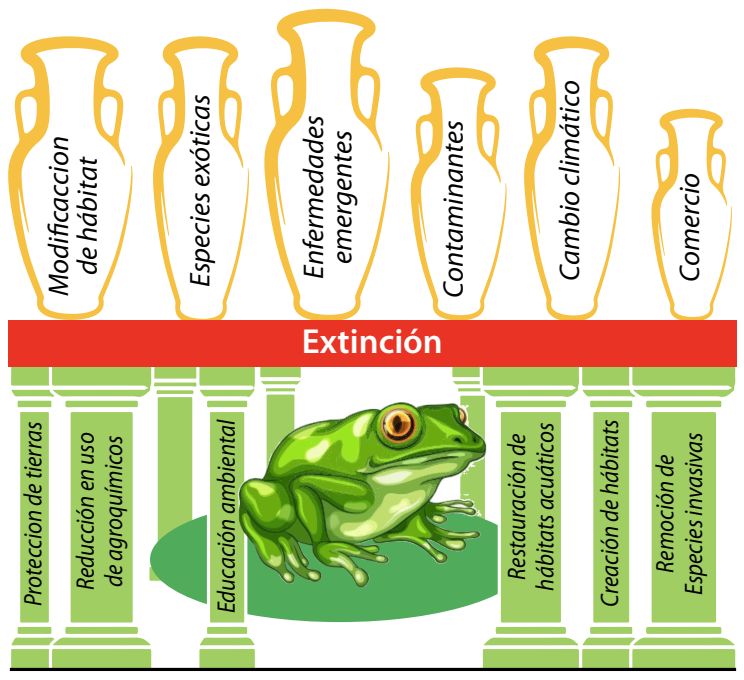

Nota. Basado en Sutherland et al., 2019

Educación ambiental: Empieza por el involucramiento y empoderamiento de las comunidades sobre sus recursos naturales, incluye laciencia ciudadana y elvoluntariado como herramientas de comunicación entre las personas y la comunidad científica. Los programas de educación ambiental sobre anfibios han demostrado ser efectivos tanto para aumentar el conocimiento sobre los anfibios como para aumentar las poblaciones.

Protección de tierras: Esta acción a mediana y pequeña escala permite solventar el problema de cambio de uso de suelo y la fragmentación. Se ha comprobado que proteger zonas de amortiguamiento de las áreas reproductivas ayuda parcialmente al aumento de las poblaciones de anfibios.

Creación de hábitats sustentables: Destinar pasturas alrededor de las ciudades, la eliminación de la vegetación perjudicial, junto con otro manejo del hábitat y el mantener o regular los niveles altos de aguas, han demostrado que pueden aumentar o mantener las poblaciones de anfibios o el número de especies de ranas. Relacionado con lo anterior, esta medida permite restaurar la conectividad del hábitat.

Restauración de hábitats acuáticos para reproducción: Se ha demostrado que la creación de pozas artificiales o semiartificiales, profundizar los estanques existentes para evitar la desecación y, en general, mejorar las condiciones de las pozas reproductivas ha aumentado las poblaciones de anfibios y el éxito reproductivo. Esta acción es muy importante ante los cambios drásticos del régimen de lluvias que ocurren actualmente.

Reducción del comercio a través de la legislación: La única acción que ha demostrado ser efectiva para evitar el comercio ilegal de especies es endurecer y mejorar la legislación en materia de tráfico de especies, a esto debería sumársele el mejorar los controles aduaneros de revisión.

Remoción directa de las especies invasoras: A pesar de muchos programas de control indirecto de especies invasoras, solo se ha demostrado que la remoción directa de la especie invasora ha logrado aumentar las poblaciones de anfibios nativos.

Translocaciones, pero no reintroducciones de cautiverio: El manejo en cautiverio es una buena estrategia para mantener vivas especies en alto riesgo, pero los programas de reintroducciones no han tenido el éxito deseado en la mayoría de los proyectos. Por el contrario, se ha demostrado que trasladar principalmente renacuajos a nuevos sitios sí es una herramienta efectiva para aumentar las poblaciones. 
Reducción en el uso de agroquímicos: Una reducción en el uso de pesticidas, fungicidas o fertilizantes demostró aumentar un poco las poblaciones de ranas. Es necesaria mayor investigación en la afectación por agroquímicos, junto con una concientización en la disminución de su uso para comprobar que esta medida es efectiva.

Investigación en curas de enfermedades: Se ha demostrado que aumentar la temperatura del agua en lugares cerrados (confinados) o el uso de antifúngicos en ambientes controlados, han sido efectivos para limpiar infecciones. No obstante, tanto estas técnicas como el uso de probióticos aún no han mostrado efectividad en vida silvestre, ya que los resultados en laboratorio aún son contradictorios.

Existen una gran cantidad de medidas que se han implementado para disminuir el embate de las extinciones de anfibios, pero no todas han funcionado, solo ciertas acciones han demostrado efectividad al aplicarse a la conservación. Así, cada una de las principales amenazas previamente descritas hacia los anfibios se puede mitigar con uno o varios pilares de la conservación (fig. 7). Los pilares se pueden aplicar de forma aislada o conjunta, por tanto al igual que la sinergia de las amenazas ha afectado a los anfibios, las estrategias de conservación deberían realizarse de forma sinérgica.

Es importante tener presente que la aplicación de cada pilar puede funcionar diferente para cada especie, género o grupo de anfibios amenazados, y se debe observar cada caso por separado. Al intentar responder al porqué han sobrevivido algunas especies, vamos a encontrar distintos panoramas así que las estrategias que apliquemos puede que funcionen mejor en algunas especies más que en otras.Al igual que rehabilitar a un enfermo convaleciente, nuestras acciones a favor de la conservación deben ser tanto cautelosas como efectivas, y la mejor forma en estos tiempos es trabajar interdisciplinariamente, en conjunto con instituciones y comunidades, a pesar de las dificultades que muchas veces implica coordinar y trabajar en diferentes estructuras sociales.

\section{Reflexiones finales}

Si una persona o un grupo de investigadores se "casa" con una especie amenazada, en cierta medida puede ser muy beneficioso: si cada estudiante de nuestras universidades se empeñara en dar un seguimiento continuo a ciertas especies amenazadas, no solo por deseo propio, sino porque las instituciones alentaran este tipo de investigación, y se tuviera una lista de las especies y proyectos en los cuales trabajar, sería más sencillo enfocar la conservación de cada especie por separado. Pero "casarse" no significa adueñarse de la especie, por el contrario, los proyectos de investigación y conservación deben compartir sus hallazgos, sus estrategias efectivas y ojalá sus recursos con otros grupos de investigadores, porque las especies no viven solas, y en el ambiente existen los ecosistemas con variedad de especies y muchas son la causas que los pueden afectar o beneficiar. Así también, quienes trabajen con pesticidas, con genética, con reproducción, entre otras disciplinas, deben recordar que si bien nuestro trabajo es importante no es el único, y solo entendiendo los múltiples factores que intervienen podemos lograr efectividad en las iniciativas.

Además, se debe trabajar en investigación proconservación y aplicar las medidas para 
mitigar la desaparición. Por lo tanto, no solo es ciencia básica lo que debemos conocer, si no ciencia aplicada al bienestar humano, al bienestar de los ecosistemas y las especies. La nueva conexión entre la investigación y la conservación debe estar cimentada en la educación ambiental, de esa forma deberíamos aprender a transformar nuestros estudios $y$ transformarlos en ciencia ciudadana, educación ambiental e involucramiento social en los proyectos de conservación e investigación. Muchos de los estudios o publicaciones sobre conservación se hallan en revistas poco accesibles al público general, por lo que es muy importante la difusión de aquellas acciones que realmente funcionen en conservación para convertirlas en información útil para tomadores de decisiones, gobierno local, funcionarios, entre otros actores.

En la conservación de anfibios podemos enfocar nuestros esfuerzos a responder una sola pregunta, y podría ser la causa principal de amenaza, pero los recursos que se asignan pueden no ser suficientes, por tanto la estrategia más efectiva es trabajar en muchos campos de combate para tener una respuesta en conjunto. De esa forma, mientras más pilares aportemos para evitar el peso de la extinción, mayores posibilidades de sobrevivencia tendrán las especies. Esta sinergia, como se mencionó, no solo se debe expresar en diferentes ramas de la ciencia, sino también con actores que puedan ser importantes en la toma de decisiones, la educación ambiental debe ir dirigida a las personas que puedan realizar cambios significativos. Debemos generar también la capacidad para integrar las amenazas y estrategias de conservación en las propuestas de futuros proyectos, ya sean financiados por las instituciones públicas o por organizaciones internacionales.
En el presente, los proyectos de investigación y conservación deben estar inmersos en el ambiente social y tratar de mostrar de qué manera las comunidades puede ayudar, ycómo ellas se pueden beneficiar de la protección del ambiente, ya que si la comunidad científica intenta en solitario proteger a los anfibios, o a cualquier otro grupo, caminará a ciegas hacia la meta. La educación ambiental, la investigación, el involucramiento de instituciones públicas y el apoyo privado nacional o internacional, son actores que brindan desde sus fortalezas una ventana que nos permiten ver una parte del problema global, así que (recordando la fábula hindú de los sabios ciegos y el elefante) mientras más personas toquen el elefante y compartan sus hallazgos, mejor podremos entender en conjunto como luce el elefante completo. 


\section{Referencias}

Altieria, A. H., Harrisona, S. B., Seemanna, J., Collina, R., Diazc, R. J., \& Knowltond, N. (2017). Tropical dead zones and mass mortalities on coral reefs. Proceedings of the National Academy of Sciences, 114, 3660-3665. https://doi.org/10.1073/pnas.1621517114

Barnosky, A., Matzke, N., Tomiya, S. et al. (2001). Has the Earth's sixth mass extinction already arrived? Nature, 471, 51-57. https://doi.org/10.1038/nature09678

Bolaños, F. (2009). Situación de los anfibios en Costa Rica. Revista Biocenosis, 22, 95-108.

Giam, X. (2017). Global biodiversity loss from tropical deforestation. Proceedings of the National Academy of Sciences, 114, 5775-5777. https://dx.doi.org/10.1073/ pnas.1706264114 IUCN. (2020). The IUCN Red List of Threatened Species. Version 2020-1. https://www. iucnredlist.org

Kerby, J. L., Hart, A., \& Storfer, A. (2012). Combined effects of virus, pesticide and predator cue on the larval tiger salamander (Ambystoma tigrinum). Ecohealth, 8, 46-54. https://doi.org/10.1007/s10393-011-0682-1

Lebboroni, M., Ricchiardino, G., Bellavita, M., \& Chelazzi G. (2006). Potential use of anurans as indicators of biological quality in upstreams of central Italy. Amphibia-Reptilia, 27, 73-79. https://doi. org/10.1163/156853806776052164

Molles, M. C. (1999). Ecology: Concepts and applications. Boston: McGraw-Hill.

Pounds, A. J., Bustamante, M. R., Coloma, L. A., Consuegra, J. A., Fogden, M. P. L., Foster, P. N., Young, B. (2006). Widespread amphibian extinctions from epidemic disease driven by global warming. Nature, 439, 28-36. https://doi.org/10.1038/nature04246

Rohr, J. R., Raffel, T. R., Romansic, J. M., Hamish-McCallum, H., \& Hudson, P. J. (2008). Evaluating the links between climate, disease spread, and amphibian declines. Proceedings of the National Academy of Science of the United States of America, 105, 1743617441. https://doi.org/10.1073/pnas.0806368105

Stuart, S. N., Chanson, J. S., Cox, N. A., Young, B. E., Rodriguez, A. S. L., Fischman, D. L., \& Waller, R. W. (2004). Status and trends of amphibian declines and extinctions worldwide. Science, 306, 1783-1786. doi:10.1126/science.1103538
Sutherland, W. J., Dicks, L. V., Ockendon, N., Petrovan, S. O., \& Smith, R. K. (2019). What Works in Conservation 2019. Cambridge, UK: Open Book Publishers, 2019. https://orcid.org/0000-0002-8304-4468

Van Rooij, P., Martel, A., Haesebrouck, F., \& Pasmans, F. (2015). Amphibian chytridiomycosis: a review with focus on fungus-host interactions. Veterinary Research, 46 (137), 1-22. https://doi.org/10.1186/ $\underline{\text { s13567-015-0266-0 }}$

Wells, K. D. (2007). The ecology and behavior of amphibians. Chicago, University of Chicago Press.

Whiles, R. M., Lips, K. R., Pringle, C. M., Kilham, S. S., Bixby, R. J., Brenes, R., Peterson, S. (2006). The effects of amphibian population declines on the structure and function of Neotropical stream ecosystems. Frontiers in Ecology and the Environment, 4, 27-34. https://doi.org/10.1890/1540-9295(2006)004[0027:TEOAPD]2.0.CO

Whitfield, S. M., Lips, K. R., \& Donnelly, M. A. (2016). Amphibian decline and conservation in Central America. Copeia, 104 (2), 351-379. https://doi. org/10.1643/CH-15-300

Wren, S., Angulo, A., Meredith, H., Kielgast, J., Dos Santos, M., \& Bishop, P. (2015). Amphibian Conservation Action Plan. IUCN SSC Amphibian Specialist Group. Recuperado de: https://wwwilucn-amphibians. org/resources/acap/ 